\title{
Retinal Image Smear as a Source of Information About Magnitude of Eye Movement
}

\author{
Leon Festinger and Jeffrey D. Holtzman \\ New School for Social Research, New York, New York
}

\begin{abstract}
A number of experiments were conducted to determine to what extent retinal image smearing during saccades provides information about the eye movement magnitude to the perceptual system. The technique involved obtaining measures of perceived movement when the total visual field was displaced in conjunction with saccadic eye movements. Trials with normal retinal smear were compared with trials on which smearing was greatly reduced or eliminated. The results are interpreted as showing that the absence of normal retinal smear during a saccade increases the uncertainty in the information available to the perceptual system and that this uncertainty results in a tendency to perceive smaller than veridical amounts of movement.
\end{abstract}

Questions concerning the source of extraretinal information about eye position available to the perceptual system, and questions concerning the accuracy of such information, have been with us at least since Helmholtz (1867/1962). Accurate information about eye position might possibly be provided by input to the perceptual system from the muscle spindles or tendon organs of the extraocular muscles. Two major kinds of observation, however, persuaded Helmholtz that this was not the case. He pointed out that if a person with a paralyzed external rectus muscle of the right eye tries to move that eye to the right (left eye occluded), he sees the visual world jump to the right. In short, he perceives as if his eye did move to the right, and since the retinal image did not change, the world must have moved. If adequate "inflow" information were available to the perceptual system from the muscles that move the

This research was supported by Grant MH-16327 from the National Science Foundation to Leon Festinger. We wish to thank Lloyd Kaufman for his helpful comments on the early draft of the article.

Requests for reprints should be sent to Leon Festinger, Psychology Department, Graduate Faculty, New School for Social Research, 65 Fifth Avenue, New York, New York 10003. eye, the perceived jumping of the visual world would not occur.

Equally persuasive is the observation that if the eye is moved mechanically, say, by tapping lightly on the sclera, the visual world is seen to jump. It is clear that the information from the extraocular muscles is not accurate enough. Helmholtz consequently proposed that the perceptual system knew about the innervation to the extraocular muscles. This "outflow information" was called "feeling of innervation" by Helmholtz, "corollary discharge" by Sperry (1950) and Teuber (1960), and "efference copy" by Von Holst and Mittelstaedt (1950).

McKay (1973) and McKay and Mittelstaedt (1974) raised the objection that, in principle, outflow information cannot be very accurate either. One argument is that the physical characteristics of the eyeball, and the muscles that move it, introduce enough error into the system so that if changes on the retina were simply compared with the monitored efferent command, the visual world would jump around whenever the eyes moved. Helmholtz (1867/1962) himself never claimed that the information provided by the "feeling of innervation" was very precise or was very strong information. Indeed, he stated the opposite: 
"As long as the field of view contains a large number of stationary objects, it is easy to be constantly aware of the degree of innervation required to hold the eye in definite positions. But when most of the objects in front of us are in motion, it is difficult to judge correctly as to rest and motion" (p. 250). And again, later on on the same page, he said that "there must be a continuous control of the amount of innervation needed to adjust the eyes and move them about, which is obtained by observing its effect on the visual images, if our judgments as to the direction of the visual axis and the objects of fixation are to be correct." Matin (1976) also argued that outflow information is not precise.

Thus, it seems that extraocular proprioceptive information is not accurate, and, perhaps, neither is information based solely on the monitored efferent commands. Possibly, the two taken together, when both sources of information are consistent with each other, might be quite accurate. There is some evidence in the literature, however, that even in combination, these two possible sources of information are rather inaccurate. Bridgeman, Hendry, and Stark (1975) asked observers to make horizontal saccades from one point to another of a patterned visual scene. The entire visual scene was made to jump periodically either to the right or the left and the observer's task was to indicate whether they saw it jump. Bridgeman et al. reported that "displacements are never detected if they occur about $10 \mathrm{msec}$ after the initiation of a saccade which is at least three times as large as the target displacement" (p. 719). If the available extraretinal information about the magnitude of the saccade were reasonably accurate, it seems likely that such relatively large displacements of the visual scene would be perceived.

It is possible, however, that some retinal information about the magnitude of a saccadic eye movement is also available, even in the absence of any stationary visual background. One possible source of retinal information is the rapid smearing of the image across the retina during a saccadic eye movement. Three studies (Brindley,
Goodwin, Kulikowski, \& Leighton, 1976; Siebeck \& Frey, 1953; Stevens et al., 1976) lead us to believe this smear may contain usable information. They all demonstrated that although the visual world is seen to jump with an attempted eye movement when the eye is partially paralyzed, this is no longer true with complete paralysis. It may be that even the small amount of retinal smearing that occurs with a partially paralyzed eye is needed for the perceptual system to assume that the intended eye movement was, in fact, executed. Although the smearing itself is not normally perceived, it could provide some additional information to the visual system about the magnitude of the saccade. If the visual world is made to jump in the middle of a saccade, as in the Bridgeman et al. study, this might interfere with the usefulness of the information contained in the retinal smear.

One can assess the usability of retinal smear information if one can break the link between magnitude of saccadic eye movement and the magnitude of smearing across the retina. For example, if one could stabilize the entire visual world on the retina as soon as a saccadic eye movement began, and maintain perfect stabilization during the saccade, one would eliminate all retinal smearing. One would want to measure if, and how much, the observer then perceived the world to jump.

In the experiments reported below, we collected data on the perceived extent of displacement, relative to the actual extent of displacement, of the entire visual field under a variety of conditions. We attempted to determine whether normal retinal smearing was an important factor in the information available to the perceptual system about the magnitude of a saccade.

\section{General Method}

\section{The Visual Display}

The visual display consisted of the outline of a square, $5^{\circ}$ by $5^{\circ}$ of visual angle, together with a spot in the center of the square. The display was generated by a Nova 2 minicomputer on a HewlettPackard Model 1310 oscilloscope equipped with a phosphor (P15) that has a decay time of less than 
$3 \mu$ sec. The display was refreshed about 1,000 times per second.

Observers viewed the display in a light-tight room. A contrast screen in front of the oscilloscope face reduced glow sufficiently to prevent the outline of the oscilloscope face from being visible except for a thoroughly dark-adapted eye. To prevent such dark adaptation, experimental sessions lasted no more than 2 minutes.

\section{Measurement of Eye Movements}

The observer's head was held fixed by a bite-board and forehead rest, and the position of the right eye (left occluded) was measured by using the doublePurkinje image "eye tracker" developed by Cornsweet and Crane (1973). The version of the eye tracker that we used has a noise level of no more than 3 minutes of arc. For each observer extensive calibration data were collected, as described in detail by Festinger, Sedgwick, and Hoitzman (1976). The output of the eye tracker was corrected for scale factor, baseline, and nonlinearities on line by the computer. Eye position data were sampled once every millisecond. The computer program also detected beginnings and ends of saccades on line, using a velocity criterion. A beginning of saccade was indicated when, over a 4-msec interval, the eye reached a velocity of $28^{\circ}$ per second. An end of saccade was indicated when the velocity then dropped below this value.

\section{Movement of the Display}

The scope control, through which the computer generated the display, contained two "fast adders," the contents of which were automatically added to the horizontal and vertical values of what was being displayed. Thus, to move the entire display to any new physical position, the only thing that had to be done was to store the appropriate values in the fast adders. Once this was done, the display was immediately painted out in the new position. In those experimental conditions in which the normal smearing of the image on the retina was interfered with, the position of the display was moved physically in space, contingent on the measured eye position, every millisecond during the saccade.

\section{Procedure and Measurement of Perceived Displacement}

The observer was asked to fixate the center spot of the display at the beginning of each trial. When a tone sounded, the observer was to move his eyes from the center spot to fixate the middle of the righthand vertical edge of the square. This tone was sounded after $2 \mathrm{sec}$ of fixation maintained within a half degree of the center spot.

The observer was told that although occasionally the display would not move, on most trials, around the time that he moved his eyes, the entire display would jump to a new position. We stated that we were interested in knowing how much the observer saw it displace. After the display jumped (or at the comparable time when it did not move), the center spot blinked on and off four times as a signal. When ready to make an estimate of the extent of displacement, the observer depressed a switch, at which time the display disappeared and the number " 0 " appeared on the scope at the position in which the center spot had last been. By the press of another switch to the right, the number would increase with an " $R$ " beside it. By the press of the switch to the left, the number would decrease and, if zero was passed, would increase with an "L" beside it. In this way, the observer was to indicate the magnitude and direction of the perceived displacement of the display.

The metric employed, it was explained, used 100 as being equal to the horizontal dimension of the square. Thus, if the square was perceived to have displaced an amount equal to, say, the distance from the center spot to the edge of the square, the correct answer would be 50 . To help the observer learn to use the scale, after the judgment was made, the correct number indicating the amount the display had actually jumped was shown. After $10 \mathrm{sec}$ of total darkness, the next trial started, with the reappearance of the square and spot in the center of the oscilloscope face. Five such trials were run in a block, with rest periods between blocks during which the observer sat in a lighted room.

\section{Manipulation of the Display During and After the Eye Movement}

Six conditions were run in which, during the saccade to the edge of the square, the retinal smearing of the display was altered in various ways. An example of each condition is presented schematically in Figure 1. The time at which the tone sounded and the interval in which the saccade took place are indicated at the bottom of the figure. All of these examples depict trials in which the eye moved accurately $\left(2.5^{\circ}\right.$ horizontally) to the right-hand edge of the square and the displacement of the square was also $2.5^{\circ}$ to the right. By displacement, we refer to the difference between the position of the square before the saccade and its position after the end of the saccade. Each condition is described below.

\section{Control Condition}

Delayed Displacement (Figure 1A). Most of the trials run were control trials in which the display remained stationary during the saccade and did not displace until 150 msec after the end of the saccadic eye movement to the edge of the square. A random number between 0 and 80 (on the measurement scale) determined the magnitude of the displacement on any trial. The example in Figure $1 \mathrm{~A}$ is for a trial in which the number 50 was chosen.

The purpose of these Delayed Displacement trials was twofold. Since the display jumped when the 
A Delayed

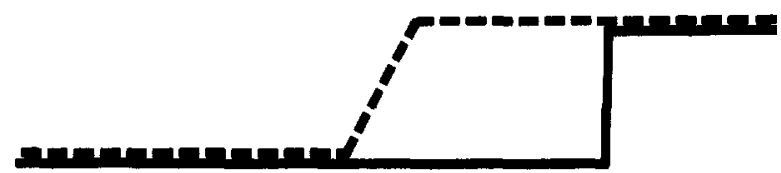

B Normal

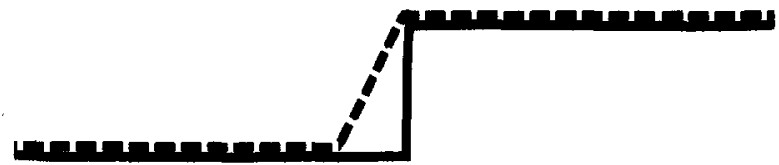

C Stable

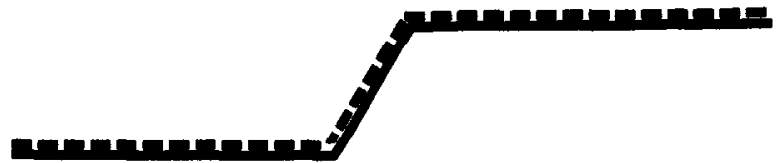

D Blank

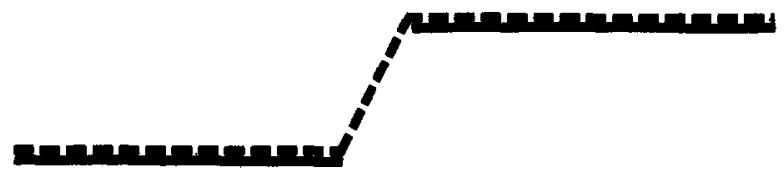

E Double

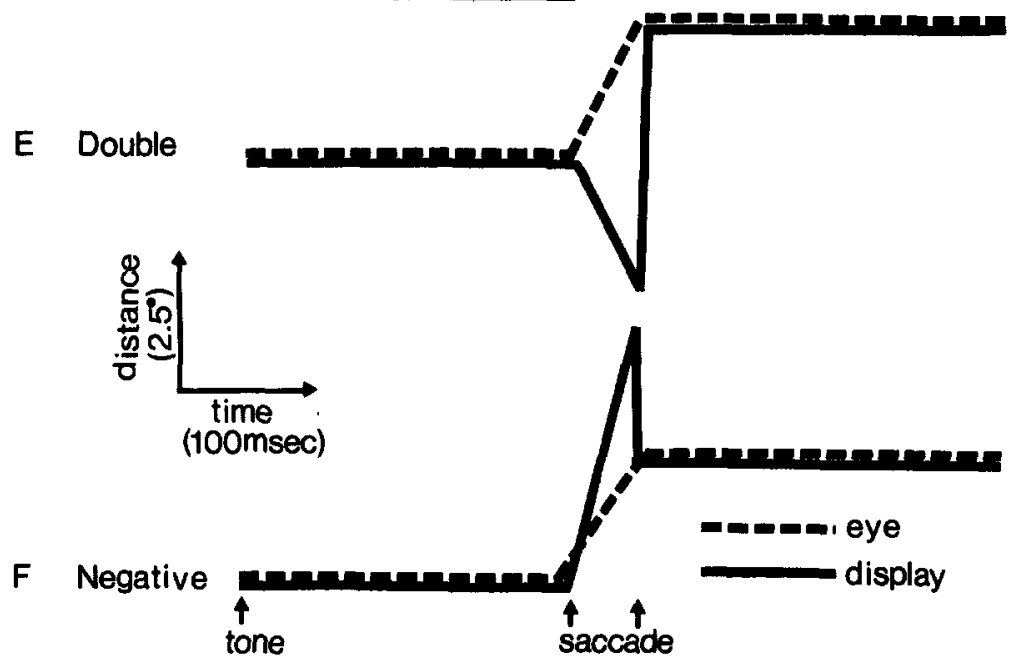

Figure 1. Time course of events in control and experimental conditions.

observer's eye was essentially stationary, perception of the amount of displacement did not require any knowledge about the amount of eye movement. Thus, the data on these trials provide a basis for assessing the perceived amount of displacement on the experimental trials. The other purpose was to continue to provide practice, with feedback about the correct answer, to help the observer to maintain accurate use of the scale of measurement.

\section{Experimental Trials}

On all experimental trials the square was presented at its end-of-saccade position as soon as the computer detected the end of the saccade, that is, slightly before the eye movement was completed. For each of the conditions described below, when the display jumped, two magnitudes of display displace- ment were used. On half of the trials the displacement was "large," equal in magnitude to the horizontal component of the saccadic eye movement (about $2.5^{\circ}$ ). On the other half of the trials the displacement was "small," one half of that magnitude.

For experimental trials, the perception of how much the square displaced necessarily depended upon knowledge of how far the eye had moved. On trials in which the displacement of the square was equal in magnitude to the eye movement, its horizontal retinal positions before and after the eye moved were identical. Any perceived change in the position of the square, thus, would be solely determined by information about change in eye position. On trials in which the displacement was half of the eye movement magnitude, any perceived change in the position of the square would be jointly deter- 
mined by its change on the retina and information concerning the magnitude of the eye movement.

For the purpose of assessing the contribution of retinal smearing to information about eye movement magnitude, experimental trials differed only in terms of what happened to the display during the saccadic eye movement. For each experimental trial, one of five conditions existed:

Normal Smear (Figure 1B). In this condition the display remained stationary for the duration of the saccade and was displaced as soon as the end of the saccade was detected. Thus, during the saccade there was normal smearing of the image on the retina, as was true in the delayed trials.

Stable (Figure $1 C$ ). In this condition we attempted to stabilize the total visual scene on the retina during the saccade by moving it, every millisecond, in accordance with the current eye position. It was destabilized and presented at its displaced position as soon as the end of saccade was detected. The ideal would have been to eliminate all retinal smearing, but this was not accomplished. The lag of 2-4 msec between the eye movement and the output of the eye tracker still left considerable smearing on the retina during the peak velocities of the saccadic eye movement. We can regard this condition as one in which the amount of retinal smearing was reduced.

Blank (Figure 1D). In this condition, as soon as the beginning of the saccade was detected, the entire display disappeared. It reappeared at its displaced position at the end of the saccade. This procedure eliminates retinal smearing.

Double Smear (Figure IE). Here, during the saccade the display was moved every millisecond an amount equal to, and in a direction opposite to, the eye movement. Thus, the smearing on the retina would, under ideal circumstances, be twice the normal amount.

Negative Smear (Figure $1 F$ ). Here, during the saccade the display was moved every millisecond in the same direction as the eye but in an amount equal to twice the eye movement. Under ideal circumstances this would have produced smearing equal in magnitude but in the opposite direction from the Normal Smear condition.

As can be seen in Figures $1 \mathrm{E}$ and $\mathrm{F}$, the displaced position of the display at the end of the saccade was the same as in the other experimental conditions.

At the beginning of the first session of data collection, 15 control trials were presented to provide practice in using the measurement scale. Thereafter, experimental trials were mixed together with control trials in the following manner. An accuracy criterion was established that required the difference between the observer's perception and the actual displacement to be less than $20 \%$ of the amount of displacement of the display. An experimental trial was run (a) if two consecutive control trials reached the criterion, (b) after three control trials if at least one of them reached the criterion, or (c) after four control trials if none reached the criterion.
Experiment 1 : Eye Movement and Display Displacement in the Same Direction

\section{Method}

Eight adult paid volunteers with normal vision were used in this experiment. None knew anything of the purposes of the study. Each observer served for about 2 hours per day on 4 consecutive days. The first session was devoted to calibration and the remaining three sessions to data collection.

For each of the five experimental conditions, trials were run in which the display did not move at all, moved a large amount (about $2.5^{\circ}$ ), or moved a smaller amount (about $1.25^{\circ}$ ). There were, then, 15 different combinations of experimental conditions. The observers always moved their eye to the right, and the display, when it displaced, also displaced to the right.

On the first day of data collection, in addition to explanation and practice, data were collected on one random permutation of the 15 experimental trials embedded among control trials. Two random permutations were run each of the next 2 days so that, in all, five measurements were made for each of the 15 kinds of trials.

On control (delayed displacement) trials, the observer was shown the correct number corresponding to the actual amount of displacement. It was not clear, however, what feedback to give to the observer on the experimental trials. Consequently, we tried various alternatives:

Some feedback. For three observers the feedback on the experimental trials told them that the "actual displacement" was within one point of the number they indicated as the perceived amount of displacement. The "correct number" displayed for two other observers was always either $25 \%$ higher or lower than the number they indicated as their perception.

No feedback. Three observers were told that sometimes they would not be shown the correct value. These three were never given any feedback on experimental trials, and feedback was omitted on one fourth of the control trials.

\section{Results}

The results, expressed as the proportion of the actual display displacement that was perceived, are shown in Table 1 for trials in which the display jumped an amount equal to the horizontal component of the eye movement and for the trials with a smaller amount of display displacement. For each trial, the amount of perceived displacement was divided by the amount that the display actually displaced. These proportions were averaged to provide the means shown in the table. The results for the trials in which the display did not 
Table 1

Mean Proportions of Actual Displacement Perceived When Display Jumps

in the Same Direction as the Eye

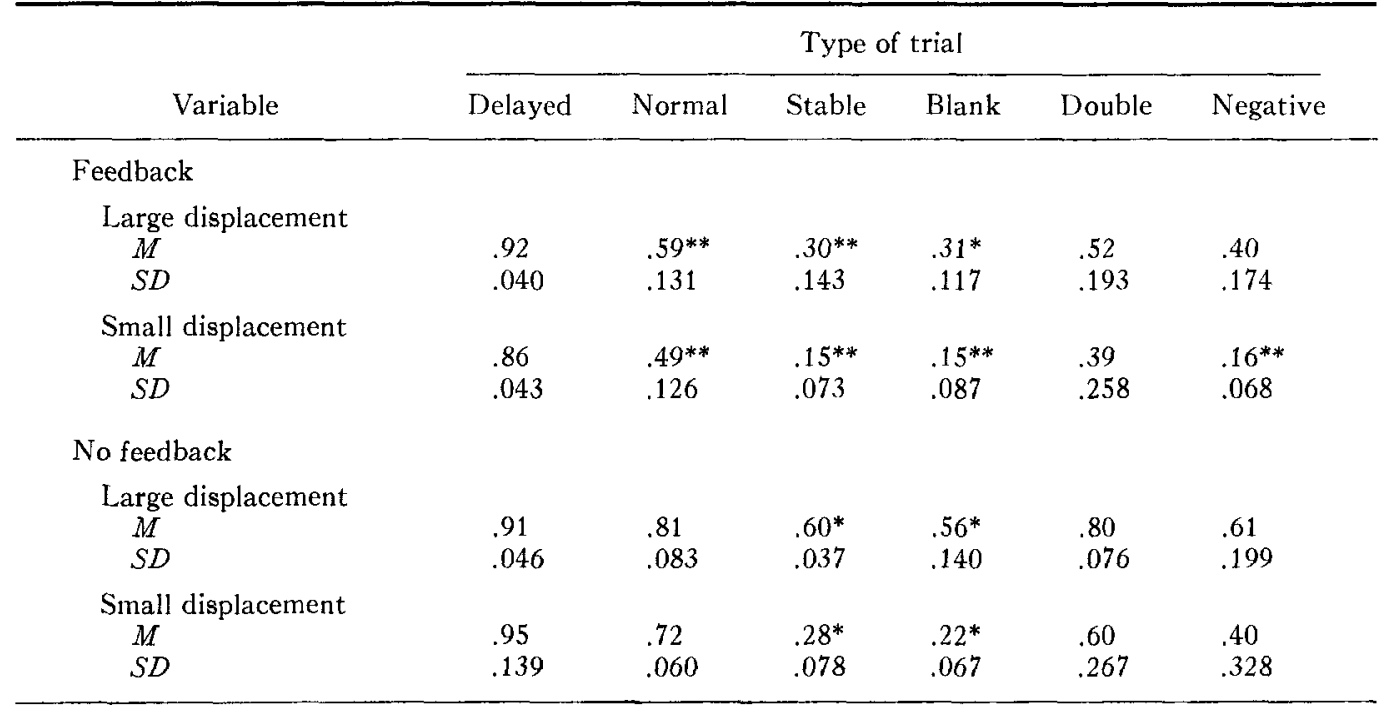

Note. Each Normal mean was tested for significance against the comparable Delayed mean. All the other means were tested against the Normal mean. Significant $p$ values are indicated where obtained. The $n$ for feedback condition $=5$; for no-feedback condition, $n=3$.

${ }^{*} p<.05$.

** $p<.01$.

change position from before to after the eye movement are not shown, since except for a very few instances, observers always perceived no displacement when no displacement occurred.

To select, for comparison, those control trials on which comparable magnitudes of display displacement occurred, we used the following procedure. For each observer, the mean and standard deviation of the horizontal eye movement magnitudes on all experimental trials were calculated. All control trials (excluding practice trials) in which the display displaced an amount falling within the range of this mean plus or minus one standard deviation were averaged for the "large displacement" proportion. All control trials falling in the range of half of the above mean plus or minus half of the standard deviation were averaged for the "small displacement" proportion.

Given the feedback concerning the true amount of displacement of the visual display on the control trials, one would expect the observers to learn to use the scale appropriately so that the perceived displacement would, on the average, be equal to the actual displacement, that is, a proportion of 1.00 . The obtained mean proportions are reasonably close to this value, but it is clear that there is a small consistent tendency to underestimate.

The main results for the experimental trials can be summarized very simply.

1. Every observer perceived less displacement on the Normal Smear trials than on the control trials.

2. Every observer perceived less displacement on the Stable and Blank trials than on the Normal Smear trials.

3. The results for the Double Smear and Negative Smear trials are a good deal less consistent from observer to observer, but on the average, the proportions for the former tend to resemble the Normal Smear results and the proportions for the latter tend to resemble the Stable and Blank results.

Another aspect of the data in Table 1 
should be noted. The average proportions obtained on the experimental trials are smaller when there was feedback than when there was no feedback. The reason for this seems clear. The perceptual experience on the experimental trials was quite different from that on the control trials. On experimental trials the perception of displacement of the display was less vivid visually and there was more uncertainty about it. This was reflected in the longer time it usually took the observers to make their judgments of the amount of displacement on the experimental trials and was also reflected in verbal reports when they were questioned at the conclusion of their last session. Since they perceived less displacement on the experimental trials and since, whatever scale number they chose was nearly "correct" (low numbers were also nearly correct in the $25 \%$ error procedure), these procedures biased them toward choosing a low number whenever they felt unsure of their perception.

Thus, for methodological reasons, it seems that the no-feedback procedure is the preferable one and the only one in which we can have some faith in the absolute, in addition to the relative, values of the obtained proportions.

\section{Discussion}

In all the experimental conditions the perception of the amount of displacement must rest, at least in part, on the availability to the perceptual system of information about how far the eye moved. In some manner, the comparison of the square's position on the retina before and after the eye movement must be evaluated with respect to such information about the eye movement. It seems abundantly clear from the data that this information about the magnitude of a saccadic eye movement is inexact. Furthermore, it seems that the normal smearing of the image across the retina that accompanies a saccade under ordinary conditions does contribute to the information about the magnitude of the eye movement. If the amount of smear is reduced (as in the Stable condition) or is removed by blanking the display during the saccade, the perceived amount of displacement of the visual display is sharply and significantly reduced.

From the data on hand thus far, it might seem that the magnitude of eye movement is underestimated by the perceptual system. Also, in the absence of retinal smear during the saccade, this underestimation is greater.

Another possible explanation of the data is that some mechanism suppresses the perception of displacement during a saccade and that, somehow, interfering with the normal retinal smear enhances this suppression. Indeed, Stark, Kong, Schwartz, Hendry, and Bridgeman (1976) proposed the existence of such a general mechanism, although they were not concerned with and did not mention retinal smearing. They reported that even if during the saccade the visual display jumps in a direction orthogonal to the eye movement, there is the same lack of perceived displacement as if it jumps in the same direction as the eye. Stark et al., however, used a slightly blurred visual stimulus, and, in addition, the maximum movement of their display was 30 minutes of arc while the observers made $10^{\circ}$ saccades. Not perceiving such a small amount of displacement relative to the eye movement is a rather different matter than the question with which we are concerned. Nevertheless, it is important to deal with this possible mechanism since, if we obtained the same results for display displacement in an orthogonal direction, it would certainly imply that our data have nothing to do with the quality of the information about eye movement that is available to the perceptual system. Experiment 2 was designed to assess this.

\section{Experiment 2: Display Displacement Orthogonal to Eye Movement}

\section{Method}

The procedure in this experiment was identical in all respects but one to the no-feedback procedure of Experiment 1. The only difference was that instead of displacement to the right (the direction of the saccade), the display was displaced upward (orthogonal to the saccade direction). Thus, the perception of the amount of displacement would not require any knowledge about the magnitude of the saccade. 
Table 2

Mean Proportion of Actual Displacement Perceived When Display Jumps Orthogonally to the Eye Movement

\begin{tabular}{lllllll}
\hline & \multicolumn{7}{c}{ Type of trial } \\
\cline { 2 - 7 } Variable & Delayed & Normal & Stable & Blank & Double & Negative \\
\hline $\begin{array}{l}\text { Large displacement } \\
M \\
S D\end{array}$ & .87 & .87 & .79 & .91 & .89 & .83 \\
$\begin{array}{l}\text { Small displacement } \\
M\end{array}$ & .087 & .070 & .041 & .156 & .051 & .227 \\
$S D$ & .92 & .65 & .68 & .56 & .78 & .51 \\
\hline
\end{tabular}

Note. Each Normal mean was tested for significance against the comparable Delayed mean. All the other means were tested against the Normal mean. No significant differences at $p<.05$ were obtained. There were three observers.

Three adult paid volunteers were observers in this experiment.

\section{Results}

Table 2 presents the data from this experiment. It is clear that the results are not at all similar to those from Experiment 1. For orthogonal displacement there is no consistent tendency for the proportions in the Normal Smear condition to be lower than the control condition.

There is also no consistent tendency at all for the proportions to be lower in the Stable and Blank conditions than in the Normal Smear condition. This indicates that the results from Experiment 1 are indeed attributable to the quality of the eye movement information. The kind of mechanism proposed by Stark et al. (1976) certainly does not explain our data.

The data in Table 2 do show one trend, although the effect is strong for only one observer. There is a tendency for the proportions in the experimental trials to be lower for smaller than for the large displacements. Part of the reason for this may be that all the observers in the orthogonal experiment developed some upward vertical component to their saccades even though they were instructed to move their eye only horizontally. Underestimating, or ignoring, this vertical component of the eye movement by the perceptual system would, of course, affect the obtained proportions more in the conditions in which the display moved less. As a matter of fact, it is interesting to note that for the two observers who showed relatively small differences between the proportions for the two magnitudes of displacement, the mean upward components of their saccades on the experimental trials are 9.3 and 8.4 minutes of arc. The observer for whom the above mentioned difference in proportions is large showed a mean upward component of 20.7 minutes of arc.

\section{Discussion}

Since we must come to the conclusion that the effects obtained in Experiment 1 are not due to some general mechanism of "suppression of movement detection," we may address the question of what is the precise effect of removing the normal retinal smear during the saccade. One interpretation is that the perceptual system consistently underestimates the magnitude of the saccade and, in the absence of retinal smearing of the image, underestimates even more. This would of course result in underestimation of the amount of displacement of the visual display only when it jumped in the same direction as the eye. It implies that if the displacement were opposite in direction to the eye movement, the magnitude of the displacement would be overestimated.

It is, hence, necessary to determine what happens in our experimental situation when, with the observer making the same 
rightward eye movement, the display jumps in the opposite direction, namely, to the left. Experiment 3 was done to investigate this issue.

Experiment 3 : Eye Movement and Display Displacement in Opposite Directions

\section{Method}

The procedure in this experiment was the identical no-feedback procedure used previously except that the visual display was displaced to the left in conjunction with the observer's rightward eye movement. Three adult paid volunteers were observers in this experiment.

\section{Results}

The results of this experiment are presented in Table 3. In the large-displacement situation there is no hint of any difference in perception among Normal Smear, Stable, and Blank conditions. There is a tendency to perceive less displacement in the Stable and Blank conditions compared with the Normal Smear condition for the smalldisplacement conditions. The differences in Table 3 are not so pronounced or consistent as in Table 1.

The most uniformly consistent finding in Table 3 is that the Negative Smear condition produced less perception of displacement than the Normal Smear condition. Why this should be the case is puzzling, particularly since in the previous two experiments this condition produced the most variable results. Our inclination is to distrust any simple interpretation of the results of the Negative Smear condition. This was the only condition that observers could distinguish as different, frequently reporting seeing flashes in peripheral vision.

Returning to the comparison of Stable, Blank, and Normal Smear conditions, it is clear that the effect of removing normal retinal smear during a saccade does not simply result in an underestimation of the magnitude of the eye movement by the perceptual system. If this were the case, displacement of the square would have been overestimated in the Stable and Blank conditions compared with the Normal Smear condition.

Before attempting an integration and explanation of the results of these three experiments, it is important to know that the results are not, somehow, an artifact of the kind of task and measurement that we employed. We, consequently, decided to do a fourth experiment in which the same underlying processes could operate with a very different perceptual task.

We can, for example, cause the display to jump at any one of many possible angles relative to the direction of eye movement, and we can ask the observer to indicate the direction in which the display displaced. Thus, for example, the observer could be asked to make a saccade to the right, and the display might displace at an angle

Table 3

Mean Proportion of Actual Displacement Perceived When Display Jumps Opposite to the Eye Movement

Type of trial

\begin{tabular}{ccccccc} 
Variable & Delayed & Normal & Stable & Blank & Double & Negative \\
\hline Large displacement & .85 & .69 & .72 & .73 & .66 & $.45^{* *}$ \\
$M$ & .051 & .147 & .189 & .066 & .127 & .123 \\
$S D$ & & & & & & \\
Small displacement & .89 & .64 & .29 & .47 & .51 & .15 \\
$M$ & .045 & .383 & .250 & .210 & .263 & .131 \\
\hline$D$ & .263 &
\end{tabular}

Note. Each Normal mean was tested for significance against the comparable Delayed mean. All the other means were tested against the Normal mean. Significant $p$ values are indicated where obtained.

$*<<.05$.

$* * p<.01$. 
of $45^{\circ}$. An underestimation of the magnitude of the eye movement would then be expected, for this angle, to yield a misperceived direction, inclined toward the vertical. We performed Experiment 4 to examine whether, with this changed task, we would obtain similar results.

\section{Experiment 4: Perceived Direction of Displacement of the Visual Scene}

\section{Method}

The general procedure in this experiment was similar to that previously reported. The same display was used, and as before, a tone was the signal for the observer to move his eye to the right-hand edge of the square. The display was made to jump to a new position, but, here, it displaced in any one of 20 possible directions. These possible directions were separated by $18^{\circ}$ and are measured counterclockwise starting at $0^{\circ}$ (direction identical with the required eye movement). Thus, the display could jump at an angle of $0^{\circ}, 18^{\circ}, 36^{\circ}, 54^{\circ}, \ldots, 306^{\circ}, 324^{\circ}, 342^{\circ}$. On half of the trials the magnitude of the displacement was 2.50 and on the other half, $1.25^{\circ}$ of visual angle. Thus there were 40 combinations of magnitude and direction of display jump.

Four conditions were included in this experiment. In the control condition the display jumped $180 \mathrm{msec}$ after the end of the saccade. In the Normal Smear, Stable, and Blank conditions the display jumped immediately on the detection of the end of the saccade. Thus, altogether, there were 160 kinds of trials combining each of the magnitude and direction combinations with each of the above four conditions.

Since the extent of displacement of the display was not contingent on the magnitude of the saccade, we placed restrictions on the permissible variation in the latter. If the actual horizontal component of the observer's eye movement was less than $1.75^{\circ}$ or greater than $3.25^{\circ}$ of visual angle, the trial was rejected and was repeated automatically later in the series. The same was the case if the vertical component of the observer's eye movement was greater than 19 minutes of arc. At the beginning of each day a random permutation of the 160 trials was chosen and presented in that order. Trials that had to be repeated were added on to the end of the permutation. Each observer served for three such days of data collection.

The method of measurement was somewhat similar to that employed in the previous experiments. When the observer depressed the switch, indicating that he was ready to make his judgment, the display disappeared, and an arrow, subtending $1^{\circ}$ of visual angle, appeared at the last physical position of the center dot. When the observer pushed another switch to the left or to the right, this arrow could be rotated counterclockwise or clockwise. The observer's task was to rotate the arrow until it pointed in the same direction in which the display had jumped. No feedback as to correctness was given on any of the trials.

Three subjects, one of them an experienced observer, participated in this experiment.

\section{Results}

For all three observers the data were symmetrical for directions of display displacement having a positive or a negative vertical component. Therefore the measurements for the latter directions of displacement were rectified by subtracting the measured perceived angle from $360^{\circ}$, and the data for upward and downward displacements were averaged together.

The perceived directions of displacement when the square jumped at angles of $0^{\circ}$, $90^{\circ}$, or $180^{\circ}$ are not, of course, important to our purposes. It suffices to say that these perceptions are reasonably veridical and there are no differences among the conditions that even approach significance. There are also negligible differences among conditions when the displacement contained only a small vertical component, that is, angles of $18^{\circ}$ and $162^{\circ}$. The results for the other angles, those with appreciable vertical components, are interesting. To simplify the presentation of the data, we averaged, for each subject, the measurements when the square displaced with a horizontal component in the same direction as the eye movement (angles of $36^{\circ}, 54^{\circ}$, and $72^{\circ}$ ) and those with a horizontal component in the direction opposite to that of the eye movement (angles of $108^{\circ}, 126^{\circ}$, and $144^{\circ}$ ).

These data are presented in Table 4 . The average measured perceived angles for the three observers are shown for the control condition. The data for the Normal Smear, Stable, and Blank conditions are presented as differences from the control condition, a positive sign denoting a difference in the direction of $90^{\circ}$, that is, a perception more vertically inclined than the perception in the control condition.

Let us first compare the results for the Normal Smear condition with the control condition. We found in previous experiments that the amount of display displacement in the same dimension (same or 
Table 4

Perceived Direction of Display Displacement In Experiment 4

\begin{tabular}{|c|c|c|c|c|}
\hline \multirow[b]{2}{*}{ Variable } & \multicolumn{4}{|c|}{$\begin{array}{l}\text { Horizontal component of display displace- } \\
\text { ment in relation to eye movement }\end{array}$} \\
\hline & $\begin{array}{l}\text { Same d } \\
\left(36^{\circ}, 54^{\circ}\right.\end{array}$ & $\begin{array}{l}\text { rection } \\
\left.\text { and } 72^{\circ}\right)\end{array}$ & $\begin{array}{l}\text { Opposit } \\
\left(108^{\circ}, 12\right.\end{array}$ & $\begin{array}{l}\text { direction } \\
\left.6^{\circ}, 144^{\circ}\right)\end{array}$ \\
\hline Magnitude of display jump & 2.50 & 1.25 & 2.50 & 1.25 \\
\hline $\begin{array}{l}\text { Delayed displacement } \\
M \text { perceived angle } \\
S D\end{array}$ & $\begin{array}{c}50.4 \\
8.26\end{array}$ & $\begin{array}{c}50.1 \\
9.72\end{array}$ & $\begin{array}{r}127.9 \\
4.00\end{array}$ & $\begin{array}{r}128.4 \\
4.06\end{array}$ \\
\hline $\begin{array}{l}\text { Normal Smear condition } \\
\quad M \\
S D\end{array}$ & $\begin{array}{r}+2.4 \\
4.56\end{array}$ & $\begin{array}{r}+4.5 \\
4.14\end{array}$ & $\begin{array}{r}+5.5 \\
2.49\end{array}$ & $\begin{array}{r}+6.6 \\
4.29\end{array}$ \\
\hline $\begin{array}{l}\text { Stable condition }{ }^{\mathrm{a}} \\
\quad M \\
S D\end{array}$ & $\begin{array}{r}+13.7 \\
4.51\end{array}$ & $\begin{array}{r}+28.8 \\
4.48\end{array}$ & $\begin{array}{r}+7.3 \\
4.18\end{array}$ & $\begin{array}{r}+3.8 \\
9.45\end{array}$ \\
\hline $\begin{array}{l}\text { Blank condition }{ }^{\mathrm{a}} \\
\quad M \\
S D\end{array}$ & $\begin{array}{r}+15.1 \\
3.32\end{array}$ & $\begin{array}{r}+32.0 \\
7.60\end{array}$ & $\begin{array}{r}+7.5 \\
2.62\end{array}$ & $\begin{array}{r}+7.6 \\
8.60\end{array}$ \\
\hline
\end{tabular}

Note. All cell entries are in degrees. There were three observers.

a In comparison with Delayed condition; + indicates a difference toward $90^{\circ}$.

opposite direction) as the eye movement was underestimated in the Normal Smear compared with the control condition. If this tendency also affects the perception of direction of movement, one would expect the data for Normal Smear to incline more toward the vertical than those for the control condition. In Table 4 there is, indeed, a small tendency for this to be the case.

Of greater interest is the comparison of the Normal Smear condition with the Stable and Blank conditions. These differences are clear and are reasonably consistent with the results obtained in the previous experiments using estimations of magnitude of displacement. When the horizontal component of the displacement was in the same direction as the eye movement, there was a large inclination to the vertical in the perceived direction of movement for the Stable and Blank conditions. They are significantly different from the Normal Smear condition, $F(2,4)=8.50, p<.05$ for $2.5^{\circ}$ and $F(2,4)=31.90, p<.01$ for $1.25^{\circ}$, respectively.

On the other hand, when the horizontal component of display movement was opposite to the direction of eye movement, there was no evidence of any difference among Normal Smear, Stable, and Blank conditions. It should be recalled that in Table 3 there was also no difference among these conditions for large display movement but there was some difference for the small movements. This latter is the only point of disagreement between the magnitude data and the direction data.

\section{General Discussion}

We can summarize the major findings from the series of studies presented in this article as follows:

1. If the entire visual scene is displaced in conjunction with a saccadic eye movement so that retinal change alone is not sufficient for veridical perception of movement (Normal Smear condition), the component of the displacement that is in the same or opposite direction to the eye movement is perceived as smaller than if the displacement occurs on a stationary retina. This is not the case, in our results, if the displacement is orthogonal to the eye movement.

2. If the normal retinal smearing during the saccade is reduced by (somewhat im- 
perfect) stabilization during the saccade, or by blanking the display during the saccade, there is large underestimation of that component of display displacement that is in the same direction as the saccade. For displacement in the opposite direction to the eye movement, this underestimation perhaps exists, but only for smaller displacements. Again, the perception of displacements orthogonal to the eye movement are not affected.

3. These findings hold whether one is measuring perceived magnitude or perceived direction of display displacement except that in the latter case there is no detectable difference at all among Normal Smear, Stable, and Blank conditions for displacements having a component in the direction opposite to the eye movement.

What conclusions can be drawn about the accuracy of extraretinal information about the magnitude of a saccadic eye movement, and what can be said about the information available from smear on the retina during a saccade?

First of all, one can say that the total combination of both possible sources of extraretinal information, together with smear, does not provide the perceptual system with very accurate information about the magnitude of a saccade. If it did, then perceptions in the Normal Smear condition would be equal to those in the Delayed condition. The finding that these two conditions do produce equivalent results for orthogonal display displacements (in which the magnitude of the saccade is irrelevant to the perception) shows that the obtained results are, indeed, dependent on the accuracy of information about the saccade.

Second, one can say that retinal smear of the image during a saccade does provide information. In the absence of such smear, perception that depends solely on extraretinal information is more erroneous. Thus, information about the magnitude of a saccade available to perception solely from extraretinal sources must be quite poor.

The findings from these experiments also specify a bit about the nature of the inaccuracy of the extraretinal information.
One cannot maintain that the extraretinal information simply underestimates the magnitude of a saccade. If that were the case, the perceived displacement of the display would indeed be underestimated when it occurred in the same direction as the eye movement, but contrary to the data, the displacement would have to be overestimated when it occurred in the opposite direction.

How, then, can one explain the findings? We think it is necessary to assume, along with McKay (1973), that the perceptual system is biased toward perceiving a stationary visual world. Even if the entire visual world is perceived to move, the bias is toward perceiving less movement. When the information about the magnitude of the eye movement is crude, as the extraretinal information may be, this bias can operate more fully.

\section{References}

Bridgeman, B., Hendry, D., \& Stark, L. Failure to detect displacement of the visual world during saccadic eye movements. Vision Research, 1975, $15,719-722$.

Brindley, G. S., Goodwin, G. M., Kulikowski, J. J., \& Leighton, D. Stability of vision with a paralysed eye. Journal of Physiology, 1976, 258, 65-66.

Cornsweet, T. N., \& Crane, H. D. Accurate twodimensional eye tracker using first and fourth Purkinje images. Journal of the Optical Society of America, 1973, 63, 921-928.

Festinger, L., Sedgwick, H. A., \& Holtzman, J. D. Visual perception during smooth pursuit eye movements. Vision Research, 1976, 16, 1377-1386.

Helmholtz, H. Handbook of physiological optics (J. P. C. Southall, trans.). New York: Dover, 1962. (Originally published, 1867.)

Matin, L. Saccades and extraretinal signal for visual direction. In R. A. Monty \& J. W. Senders (Eds.), Eye movements and psychological processes. Hillsdale, N.J. : Erlbaum, 1976.

McKay, D. M. Visual stability and voluntary eye movements. In R. Jung (Ed.), Central processing of visual information. New York: Springer, 1973.

McKay, D. M., \& Mittelstaedt, S. Visual stability and motor control (reafference revisited). In W. D. Keidel (Ed.), Cybernetics and bionics, Munich: Oldenbourg, 1974.

Siebeck, R., \& Frey, R. Die Wirkungen Muskelerschlaffender Mittel auf die Augenmuskeln. Anaesthesist, 1953, 2, 138-141.

Sperry, R. W. Neural basis of the spontaneous optokinetic response produced by visual neural inversion. Journal of Comparative and Physiological Psychology, 1950, 43, 482-489. 
Stark, L., Kong, R, Schwartz, S., Hendry, D., \& Bridgeman, B. Saccadic suppression of image displacement. Vision Research, 1976, 16, 11851187.

Stevens, J. K., Emerson, R. C., Gerstein, G. L., Kallos, T., Neufeld, G. R., Nicholas, C. W., \& Rosenquist, A. C. Paralysis of the awake human: Visual perceptions. Vision Research, 1976, 16, 93-98.
Teuber, H. L. Perception. In J. Field (Ed.), Handbook of Physiology-Neurophysiology III. Baltimore: Williams \& Wilkins, 1960.

Von Holst, E., \& Mittelstaedt, H. Das Reafferenzprinzip (wechselwirkungen zwischen Zentralnervensystem und Peripherie). Naturwissenschaften, 1950, 37, 464-476.

Received May 30, 1978 